\title{
Rational Optimization of the Binding Affinity of CD4 \\ Targeting Peptidomimetics With Potential Anti HIV Activity
}

Axel T. Neffe*, Matthias Bilang, llona Grüneberg, and Bemd Meyer

Supporting Information

\section{Table of Contents}

a) Table specifying the purity of the new compounds 3-11 (page S2)

b) Chemicals, yields, and characterization of the peptidomimetic s $\mathbf{3 - 1 1} .\left({ }^{1} \mathrm{H}\right.$ and ${ }^{13} \mathrm{C}$ NMR data, HPLC chromatograms of $\mathbf{3}, \mathbf{4}, \mathbf{6 - 8}, \mathbf{1 0}, \mathbf{1 1}$ and ${ }^{1} \mathrm{H}$ NMR spectra of 5 and 9) (pagesS3-S12)

c) Surface plasmon resonance experimental details and data points fitted to a one-site binding model of $\mathbf{3}$ and 6-11 (pages S13-S15)

d) STD NMR experimental details and spectra of 6. (page S16-S17)

e) Prona se digestion assa y experimental details (page S18)

f) Details of the docking procedure. (page S 19)

g) references (page S20) 


\section{Compound No. Punity}

3

$99.0^{a, b}$

4

$98.0^{\mathrm{a}, \mathrm{b}}$

5

$94.3^{c}$

6

$95.7^{\mathrm{a}}$

7

$98.1^{\mathrm{a}}$

8

$96.6^{\mathrm{a}}$

9

$96.1^{c}$

10

$98.5^{\mathrm{a}}$

11

$97.4^{\mathrm{a}, \mathrm{b}}$

Table 1: Purity of the peptidomimetics 3-12 a: as determined by HPLC. $b$ : as sum of integrals of the peaks of the protonated and unprotonated form of the peptidomimetics [therefore 2 peaks are present in the respective chromatogram, the corresponding MALDI TOF MS spectra show only one compound]. c: purity determined from the: ${ }^{1} \mathrm{H}$ NMR spectrum. 
b)Chemic als, yields and characterization of the peptidomimetics

Chemicals: All isocyanates and cyclopropyl isothioc yanate were purchased from Lanc aster Synthesis. Prolinol substituted 2' chloro trityl resin and all a mino acid derivatives are from ACT. TBTU was from Fluka. DSS, all salts, ac etic a nhydride and TFA a re from Merck. DMF was purc hased from Proligo, Acetonitril from Baker, and 20\% Piperidin in DMF solution from PE Biosystems. 8'-quinolyl oxyacetic acid was prepared as described before. ${ }^{[1]}$

\section{Compound 3}

Compound 3 was synthesized in ca. $0.3 \%$ yield over all steps $(0.1 \mathrm{mg}, 0.1 \mu \mathrm{mol})$ 3 is a white solid, MALDI-TOF-MS: $782\left(\mathrm{M}+\mathrm{H}^{+}\right), 804\left(\mathrm{M}+\mathrm{Na}^{+}\right), 820\left(\mathrm{M}+\mathrm{K}^{+}\right)$.

The yield in the synthesis of this compound was probably due to the lower activity of the thioisocyanate compared to the isocyanates. The binding affinity of $\mathbf{3}$ has been determined by SPR, but because of the low amount it was not characterized by NMR.

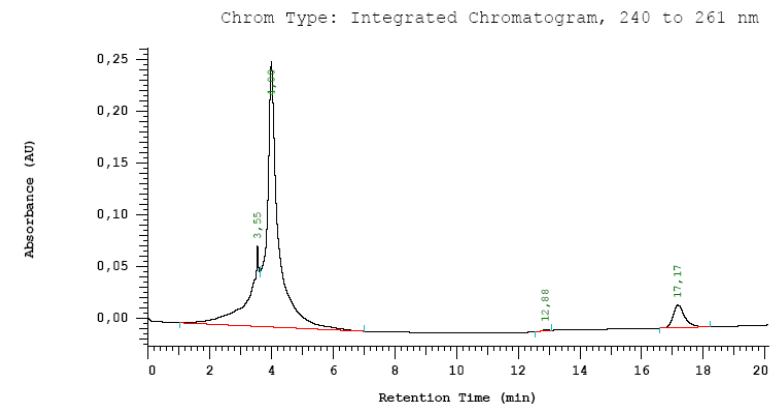

Figure 1: HPLC chromatogram of 3. Two peaks are present in the chromatogram due to elution of the protonated and unprotonated species. Only the mass of the desired product is detectable in the MALDI-TOF. 


\section{Compound 4}

Compound 4 was synthesized in ca. $1 \%$ yield over all steps $(0.7 \mathrm{mg}, 0.9 \mu \mathrm{mol})$

4 is a white solid, MALDI-TOF-MS: $770\left(\mathrm{M}+\mathrm{H}^{+}\right), 792\left(\mathrm{M}+\mathrm{Na}^{+}\right), 808\left(\mathrm{M}+\mathrm{K}^{+}\right)$.

NMR data: $500 \mathrm{MHz}, \mathrm{T}=295 \mathrm{~K}, \mathrm{pH}=2, \mathrm{H}_{2} \mathrm{O} / \mathrm{D}_{2} \mathrm{O} 9: 1$, calibrated to $\mathrm{HDO}=4.725 \mathrm{ppm}$ and $\mathrm{MeOH}=48.372 \mathrm{ppm}$

\begin{tabular}{|c|c|c|c|c|c|}
\hline Spin System & $\mathrm{NH}$ & $H \alpha / \alpha^{\prime}$ & $H \beta / \beta^{\prime}$ & $\mathrm{H} \gamma / \gamma^{\prime}$ & Miscellaneous \\
\hline$\beta$-NOA & & & & & $\begin{array}{l}\text { H1: 7.178, H3: 7.219, H4: 7.829, H5: } \\
\text { 7.816, H6: 7.366, H7: 7.453, H8: } \\
\text { 7.758, O-CH2: } 4.776\end{array}$ \\
\hline Lys & 8.404 & 4.368 & $1.695 / 1.604$ & 1.053 & 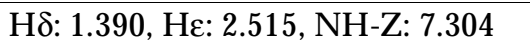 \\
\hline Val & 8.145 & 3.988 & 1.909 & 0.778 & \\
\hline Gly & 8.456 & 3.878 & & & \\
\hline Thr & 7.921 & 4.453 & 3.958 & 1.066 & \\
\hline $\begin{array}{l}\text { Prolinol } \\
\text { tButyl }\end{array}$ & $(8.013)$ & 4.148 & $1.906 / 1.795$ & $1.952 / 1.818$ & $\begin{array}{l}\mathrm{Hd} / \mathrm{d}^{\prime}: 3.648 / 3.508, \mathrm{H} 1 / 1^{\prime}: \\
4.196 / 4.177 \\
\text { tBu: } 1.155\end{array}$ \\
\hline
\end{tabular}

Table $2:{ }^{1} \mathrm{H} N M R$ chemical shifts of the trans rotamer of 4 (determined by ${ }^{1} \mathrm{H}, \mathrm{TOCSY}, \mathrm{ROESY}, \mathrm{COSY}, \mathrm{HSQC}$ ).

\begin{tabular}{|l|l|l|l|l|}
\hline Spin System & $\mathrm{C} \alpha$ & $\mathrm{C} \beta$ & $\mathrm{C} \gamma$ & Miscellaneous \\
\hline $\boldsymbol{\beta}$-NOA & & & & $\begin{array}{l}\mathrm{C} 1: 106.91, \mathrm{C} 3: 117.512, \mathrm{C} 4: 126.854, \mathrm{C} 5: 126.682, \mathrm{C} 6: \\
124.39, \mathrm{C} 7: 126.739, \mathrm{C} 8: 126.682, \mathrm{O}-\mathrm{CH}_{2}: ?\end{array}$ \\
\hline Lys & 52.959 & 29.484 & 21.516 & $\mathrm{Cd}: 25.807, \mathrm{Ce}: 39.012$ \\
Val & 59.051 & 29.561 & 18.006 & \\
\hline Gly & 41.938 & & & \\
\hline Thr & 56.724 & 66.923 & 17.783 & \\
\hline Prolinol & 56.108 & 26.197 & 23.522 & $\mathrm{Cd}: 47.277, \mathrm{C} 1: 62.542$ \\
\hline $\boldsymbol{t}$ Butyl & & & & tBu: 27.255 \\
\hline
\end{tabular}

Table 3: ${ }^{13} \mathrm{C}$ NMR chemical shifts of the trans rotamer of 4 (determined from the HSQC).

\begin{tabular}{|c|c|c|c|c|c|}
\hline Spin System & $N \mathrm{H}$ & $H \alpha / \alpha^{\prime}$ & $\mathbf{H} \beta / \beta^{\prime}$ & $\mathbf{H} \gamma / \gamma^{\prime}$ & Miscellaneous \\
\hline \multicolumn{6}{|l|}{$\beta-N O A$} \\
\hline Lys & 8.438 & 4.355 & $1.701 / 1.604$ & 1.053 & 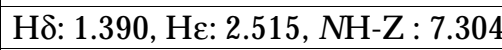 \\
\hline Val & 8.059 & 4.043 & 1.916 & 0.778 & \\
\hline Gly & 8.456 & 3.915 & & & \\
\hline Thr & 7.797 & 4.942 & 3.988 & 1.059 & \\
\hline \multicolumn{6}{|l|}{ Prolinol } \\
\hline$t \mathrm{Bu}$ & 1.098 & & & & \\
\hline
\end{tabular}

Tabelle 4: ${ }^{1} \mathrm{H}$ NMR chemical shifts of the cis rotamer of 4 (determined by ${ }^{1} \mathrm{H}$, TOCSY, ROESY, COSY, HSQC).

No individual ${ }^{13} \mathrm{C}$ NMR chemical shifts could be determined for the cis rotamer. 


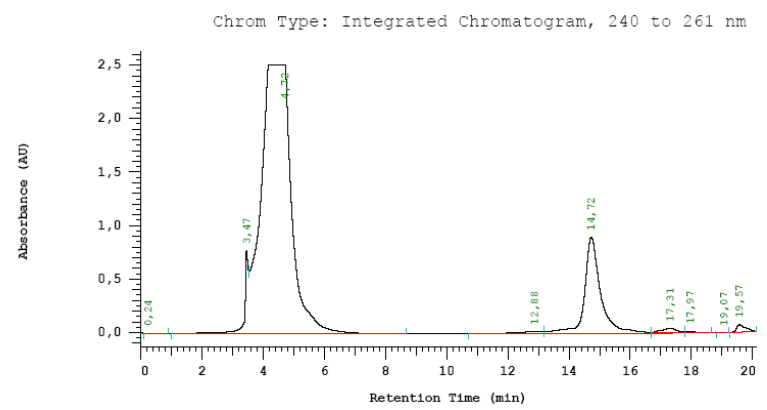

Figure 2: HPLC chromatogram of 4. Two peaks are present in the chromatogram due to elution of the protonated and unprotonated species. Only the mass of the desired product is detectable in the MALDI-TOF.

\section{Compound 5}

Compound 5 was synthesized in ca. $1 \%$ yield over all steps $(0.7 \mathrm{mg}, 0.9 \mu \mathrm{mol})$. 5 is a white solid, MALDI-TOF-MS: $782\left(\mathrm{M}+\mathrm{H}^{+}\right), 804\left(\mathrm{M}+\mathrm{Na}^{+}\right), 820\left(\mathrm{M}+\mathrm{K}^{+}\right)$.

NMR data: $500 \mathrm{MHz}, \mathrm{T}=290 \mathrm{~K}, \mathrm{MeOH}-\mathrm{d} 4$, calibrated to $\mathrm{CD}_{2} \mathrm{H}-\mathrm{OD}=3.34 \mathrm{ppm}$ and $47.84 \mathrm{ppm}$

\begin{tabular}{|c|c|c|c|c|}
\hline Spin System & $H \alpha / \alpha^{\prime}$ & $H \beta / \beta^{\prime}$ & $\mathrm{H} \gamma / \gamma^{\prime}$ & Miscellaneous \\
\hline$\beta-N O A$ & & & & $\begin{array}{l}\mathrm{H} 1: \text { 7.281, H3: 7.310, H4: 7.836, H5: } \\
\text { 7.831, H6: 7.390, H7: 7.475, H8:7.797, } \\
\text { O-CH}_{2}: 4.758\end{array}$ \\
\hline Lys & 4.616 & $1.886 / 1.766$ & 1.356 & 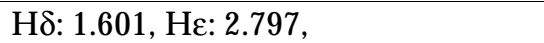 \\
\hline Val & 4.117 & 2.066 & $1.011 / 0.996$ & \\
\hline Gly & $4.069 / 3.833$ & & & \\
\hline Thr & 4.565 & 4.076 & 1.201 & \\
\hline Prolinol & 4.266 & Ca. 2.01 & $2.088 / 1.961$ & $\begin{array}{l}\mathrm{H} \delta / \delta^{\prime}: 3.819 / 3.638, \mathrm{H} 1 / 1^{\prime}: \\
4.361 / 4.015\end{array}$ \\
\hline Cyclopentyl & & & & $\begin{array}{l}\text { H1: 3.872, H2/2': 1.911/1.464, } \\
\text { H3/3': } 1.607 / 1.360\end{array}$ \\
\hline
\end{tabular}

Table 5: ${ }^{1} \mathrm{H}$ NMR chemical shifts of 5 (determined by ${ }^{1} \mathrm{H}$, TOCSY, ROESY, COSY, HSQC).

\begin{tabular}{|l|l|l|l|l|}
\hline Spin System & $\mathrm{C} \alpha$ & $\mathrm{C} \beta$ & $\mathrm{C} \gamma$ & Miscellaneous \\
\hline$\beta-N O A$ & & & & $\begin{array}{l}\mathrm{C} 1: 107.056, \mathrm{C} 3: 118.280, \mathrm{C} 4: 126.869, \mathrm{C} 5: 126.869, \mathrm{C} 6: \\
123.915, \mathrm{C} 7: 126.233, \mathrm{C} 8: 126.788, \mathrm{O}-\mathrm{CH}_{2}: 66.847\end{array}$ \\
\hline Lys & 52.357 & 31.388 & 21.980 & $\mathrm{C} 8: 23.271, \mathrm{C} \varepsilon: 39.105$ \\
\hline Val & 59.930 & 30.266 & 17.992 & \\
\hline Gly & 41.944 & & & \\
\hline Thr & 57.436 & 67.029 & & \\
\hline Prolinol & 56.522 & 26.720 & 30.167 & $\mathrm{C} 8:$ ca. 50, C1: 63.384 \\
\hline Cyclopentyl & & & & $\mathrm{C} 1: 52.571, \mathrm{C} 2 / 5: 32.284, \mathrm{C} 3 / 4: 26.710$ \\
\hline
\end{tabular}

Table 6: ${ }^{13} \mathrm{C}$ NMR chemical shifts of 5 (determined from the HSQC).

With methanol as solvent, no cis/trans rotamers could be identified. 


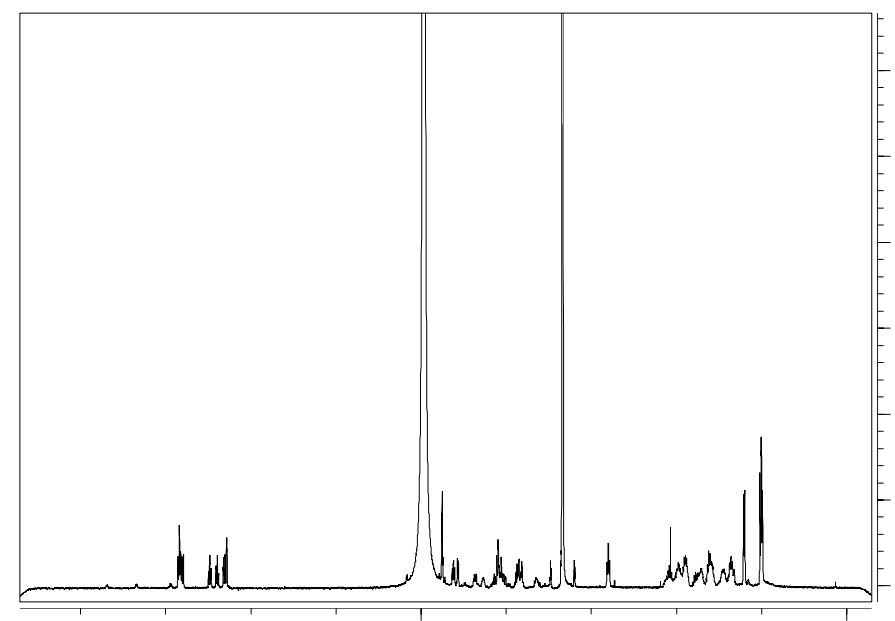

Figure 3: ${ }^{1} \mathrm{H} N M R$ spectrum of 5 in $\mathrm{CD}_{3} \mathrm{OD}$.

\section{Compound 6}

Compound 6 was synthesized in $6 \%$ yield over all steps $(4.5 \mathrm{mg}, 5.6 \mu \mathrm{mol})$

6 is a white solid, MALDI-TOF-MS: $800\left(\mathrm{M}+\mathrm{H}^{+}\right), 822\left(\mathrm{M}+\mathrm{Na}^{+}\right), 838\left(\mathrm{M}+\mathrm{K}^{+}\right)$.

NMR data: $500 \mathrm{MHz}, \mathrm{T}=290 \mathrm{~K}, \mathrm{pH}=2, \mathrm{H}_{2} \mathrm{O} / \mathrm{D}_{2} \mathrm{O}$ 9:1, calibrated to $\mathrm{HDO}=4.88 \mathrm{ppm}$ and Lys-Ce $=38.567$.

\begin{tabular}{|c|c|c|c|c|c|}
\hline Spin System & $\mathrm{NH}$ & $H \alpha / \alpha^{\prime}$ & $H \beta / \beta^{\prime}$ & $\mathbf{H} \gamma / \gamma^{\prime}$ & Miscellaneous \\
\hline$\beta$-NOA & & & & & $\begin{array}{l}\text { H1: 7.264, H3: 7.304, H4: } \\
\text { 7.915, H5: 7.905, H6: 7.46, H7: } \\
\text { 7.543, H8: 7.848, O-CH} 2: 4.854\end{array}$ \\
\hline Lys & 8.523 & 4.454 & 1.746 & 1.149 & 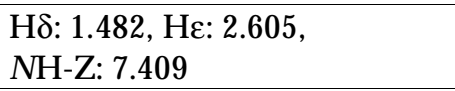 \\
\hline $\begin{array}{l}\text { Val } \\
\text { Gly }\end{array}$ & $\begin{array}{l}8.284 \\
8.594\end{array}$ & $\begin{array}{l}4.082 \\
3.966\end{array}$ & 1.997 & 0.889 & \\
\hline Thr & 8.039 & 4.547 & 4.047 & 1.158 & \\
\hline Prolinol & & 4.263 & $2.034 / 1.91$ & $2.004 / 1.917$ & $\begin{array}{l}\mathrm{H} \delta / \delta^{\prime}: 3.761 / 3.645, \mathrm{H} 1 / 1^{\prime}: \\
4.371 / 4.065\end{array}$ \\
\hline Ethoxycarbonylmethyl & 7.343 & & & & $\begin{array}{l}\mathrm{N}-\mathrm{CH}_{2}: 3.862, \mathrm{O}-\mathrm{CH}_{2}: 4.177, \\
\mathrm{CH}_{3}: 1.232\end{array}$ \\
\hline
\end{tabular}

Table 10: ${ }^{1} \mathrm{H}$ NMR chemical shifts of the trans rotamer of $\mathbf{6}$ (determined by ${ }^{1} \mathrm{H}, \mathrm{TOCSY}, \mathrm{ROESY}, \mathrm{COSY}, \mathrm{HSQC}$ ).

\begin{tabular}{|l|l|l|l|l|}
\hline Spin System & $\mathrm{C} \alpha$ & $\mathrm{C} \beta$ & $\mathrm{C} \gamma$ & Miscellaneous \\
\hline$\beta-N O A$ & & & & $\begin{array}{l}\mathrm{C} 1: 106.579, \mathrm{C} 3: 117.979, \mathrm{C} 4: 127.28, \mathrm{C} 5: 127.203, \mathrm{C} 6: \\
124.163, \mathrm{C} 7: 126.478, \mathrm{C} 8: 126.443, \mathrm{O}-\mathrm{CH}_{2}: 66.162\end{array}$ \\
\hline Lys & 52.54 & 29.823 & 26.664 & $\mathrm{C} 8: 26.571, \mathrm{C} \varepsilon: 38.567$ \\
\hline Val & 59.368 & 29.356 & 17.463 & \\
\hline Gly & 41.82 & & & \\
\hline Thr & 56.931 & 64.1 & 17.956 & \\
\hline Prolinol & 56.065 & 23.913 & 26.651 & $\mathrm{C} \delta: 48.296, \mathrm{C} 1: 64.552$ \\
\hline $\begin{array}{l}\text { Ethoxycarbony } \\
\text { lmethyl }\end{array}$ & & & & $\mathrm{N}-\mathrm{CH}_{2}: 41.949, \mathrm{O}_{2}: 62.031, \mathrm{CH}_{3}: 12.767$ \\
\hline
\end{tabular}

Table 11: ${ }^{13} \mathrm{C}$ NMR chemical shifts of the trans rotamer of 6 (determined from the HSQC). 


\begin{tabular}{|l|l|l|l|l|l|}
\hline Spin System & $\mathbf{N H}$ & $\mathbf{H} \alpha / \boldsymbol{\alpha}^{\prime}$ & $\mathbf{H} \beta / \boldsymbol{\beta}^{\prime}$ & $\mathbf{H} \gamma / \gamma^{\prime}$ & Miscellaneous \\
\hline$\beta-N O A$ & & & & & \\
\hline Lys & 8.549 & 4.439 & 1.741 & 1.142 & $\begin{array}{l}\mathrm{H} \delta: 1.489, \mathrm{H} \varepsilon: 2.595, \\
\text { NH-Z: 7.409 }\end{array}$ \\
\hline Val & 8.203 & 4.099 & 1.991 & 0.866 & \\
\hline Gly & 8.506 & 3.982 & & & \\
\hline Thr & 7.941 & 5.018 & 4.064 & 1.164 & \\
\hline Prolinol & & & & & $\mathrm{N}-\mathrm{CH}_{2}: 3.862$ \\
\hline $\begin{array}{l}\text { Ethoxycarbo } \\
\text { nylmethyl }\end{array}$ & 7.035 & & & & \\
\hline
\end{tabular}

Table 12: ${ }^{1} \mathrm{H}$ NMR chemical shifts of the cis rotamer of $\mathbf{6}$ (determined by ${ }^{1} \mathrm{H}, \mathrm{TOCSY}, \mathrm{ROESY}, \mathrm{COSY}, \mathrm{HSQC}$ ).

No individual ${ }^{13} C$ NMR chemical shifts could be determined for the cis rotamer.

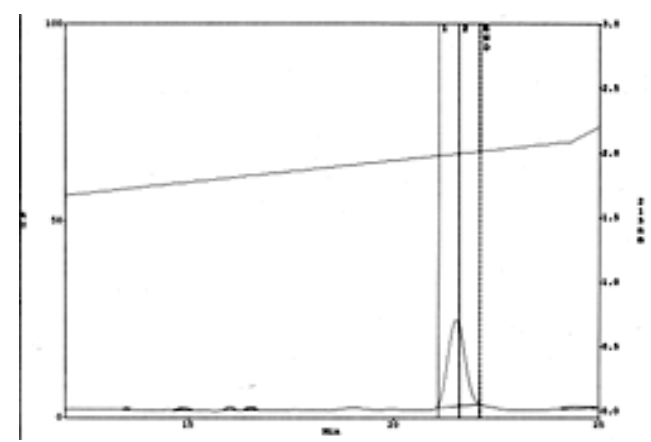

Figure 4 HPLC chromatogram of 6.

Compound 7

Compound 7 was synthesized in $3.6 \%$ yield over all steps $(1.4 \mathrm{mg}, 1.8 \mu \mathrm{mol})$ 7 is a white solid, MALDI-TOF-MS: $771\left(\mathrm{M}+\mathrm{H}^{+}\right), 793\left(\mathrm{M}+\mathrm{Na}^{+}\right), 809\left(\mathrm{M}+\mathrm{K}^{+}\right)$.

NMR data: $700 \mathrm{MHz}, \mathrm{T}=285 \mathrm{~K}, \mathrm{pH}=2, \mathrm{H}_{2} \mathrm{O} / \mathrm{D}_{2} \mathrm{O}$ 9:1, calibrated to $\mathrm{HDO}=4.7 \mathrm{ppm}$ and Lys-C $\varepsilon=38.943 \mathrm{ppm}$.

\begin{tabular}{|c|c|c|c|c|c|}
\hline Spin System & $\mathrm{NH}$ & $H \alpha / \alpha^{\prime}$ & $H \beta / \beta^{\prime}$ & $\mathrm{H} \gamma / \gamma^{\prime}$ & Miscellaneous \\
\hline ChinOA & & & & & $\begin{array}{l}\mathrm{H} 2: 8.891, \mathrm{H} 3: 7.866, \mathrm{H} 4: 8.830, \mathrm{H} 5: \\
\text { 7.680, H6: 7.631, H7: 7.294, O-CH} \text { : } \\
\text { 4.843 }\end{array}$ \\
\hline Lys & 8.683 & 4.234 & $1.619 / 1.570$ & 1.186 & 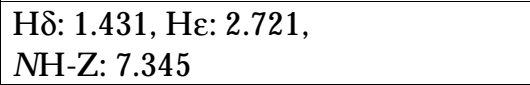 \\
\hline Val & 8.294 & 3.883 & 1.861 & 0.684 & \\
\hline $\begin{array}{l}\text { Gly } \\
\text { Thr }\end{array}$ & $\begin{array}{l}8.465 \\
7.896\end{array}$ & $\begin{array}{l}3.743 \\
4.314\end{array}$ & 3.831 & 0.909 & \\
\hline Prolinol & & 3.996 & $1.749 / 1.645$ & $1.805 / 1.667$ & $\begin{array}{r}\mathrm{H} \delta / \delta^{\prime}: 3.512 / 3.373 \\
\mathrm{H} 1 / 1^{\prime}: 4.031 / 3.647\end{array}$ \\
\hline tert.Butyl & n.b. & & & & $t \mathrm{Bu}: 0.987$ \\
\hline
\end{tabular}

Table 13: ${ }^{1} \mathrm{H}$ NMR chemical shifts of the trans rotamer of 7 (determined by ${ }^{1} \mathrm{H}, \mathrm{TOCSY}, \mathrm{ROESY}, \mathrm{COSY}, \mathrm{HSQC}$ ). 


\begin{tabular}{|c|c|c|c|c|}
\hline Spin System & $\mathrm{C} \alpha$ & $C \beta$ & $\mathrm{C} \gamma$ & Miscellaneous \\
\hline $\begin{array}{l}\text { ChinOA } \\
\text { Lys }\end{array}$ & 52.949 & 29.535 & 21.32 & $\begin{array}{l}\text { C2: 146.694, C3: 121.543, C4: 143.514, C5: 121.013, C6: } \\
\text { 129.259, C7: 114.063, O-CH }: 66.560 \\
\text { C } 8: 25.905, \text { C } \varepsilon: 38.943\end{array}$ \\
\hline Val & 58.776 & 29.583 & 17.739 & \\
\hline Gly & 41.296 & & & \\
\hline Thr & 56.436 & 67.038 & 17.834 & \\
\hline Prolinol & 56.054 & 26.001 & 22.514 & C8: 47.218, C1: 62.501 \\
\hline tert.Butyl & & & & t.Bu: 27.386 \\
\hline
\end{tabular}

Table 14: ${ }^{13} \mathrm{C}$ NMR chemical shifts of the trans rotamer of $\mathbf{7}$ (determined from the HSQC).

\begin{tabular}{|l|l|l|l|l|l|}
\hline Spin System & $\mathbf{N H}$ & $\mathbf{H} \alpha / \boldsymbol{\alpha}^{\prime}$ & $\mathbf{H} \beta / \boldsymbol{\beta}^{\prime}$ & $\mathbf{H} \gamma / \gamma^{\prime}$ & Miscellaneous \\
\hline ChinOA & & & & & \\
\hline $\begin{array}{l}\text { Lys } \\
\text { Val }\end{array}$ & 8.204 & 3.921 & 1.823 & 0.697 & \\
\hline Gly & & 3.786 & & & \\
\hline Thr & 7.749 & 4.829 & 3.857 & 0.915 & \\
\hline Prolinol & $\mathrm{XXX}$ & & & & $\mathrm{XXX}$ \\
\hline tert.Butyl & & & & & $t \mathrm{Bu}: 27.29$ \\
\hline
\end{tabular}

Table 15: ${ }^{1} \mathrm{H}$ NMR chemical shifts of the cis rotamer of $\mathbf{7}$ (determined by ${ }^{1} \mathrm{H}$, TOCSY, ROESY, COSY, HSQC).

No individual ${ }^{13} \mathrm{C}$ NMR chemical shifts could be determined for the cis rotamer.

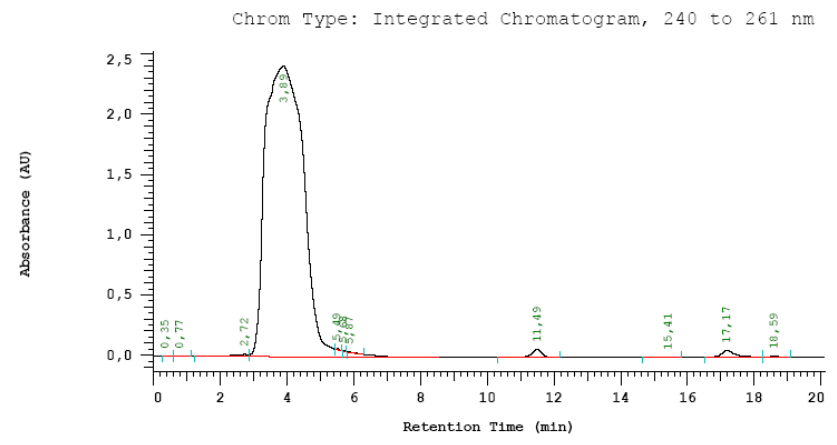

Figure 5: HPLC chromatogram of 7.

\section{Compound 8}

Compound 8 was synthesized in $3.5 \%$ yield over all steps $(1.3 \mathrm{mg}, 1.7 \mu \mathrm{mol})$ 8 is a white solid, MALDI-TOF-MS: $745\left(\mathrm{M}+\mathrm{H}^{+}\right), 767\left(\mathrm{M}+\mathrm{Na}^{+}\right), 783\left(\mathrm{M}+\mathrm{K}^{+}\right)$.

NMR data: $700 \mathrm{MHz}, \mathrm{T}=285 \mathrm{~K}, \mathrm{pH}=2, \mathrm{H}_{2} \mathrm{O} / \mathrm{D}_{2} \mathrm{O}$ 9:1, calibrated to $\mathrm{HDO}=4.7 \mathrm{ppm}$ and Lys-C $\varepsilon=38.978 \mathrm{ppm}$.

\begin{tabular}{|c|c|c|c|c|c|}
\hline Spin System & $\mathrm{NH}$ & $H \alpha / \alpha^{\prime}$ & $H \beta / \beta^{\prime}$ & $H \gamma / \gamma^{\prime}$ & Miscellaneous \\
\hline ChinOA & & & & & $\begin{array}{l}\mathrm{H} 2: 8.896, \mathrm{H} 3: 7.865, \mathrm{H} 4: 8.865, \mathrm{H} 5: \\
\text { 7.680, H6: 7.634, H7: 7.289, O-CH} \text { : } \\
4.855\end{array}$ \\
\hline Lys & 8.679 & 4.240 & 1.601 & 1.182 & 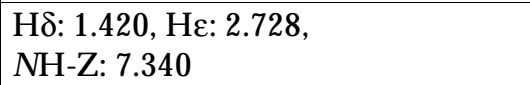 \\
\hline Val & 8.270 & 3.901 & 1.817 & 0.685 & \\
\hline Gly & 8.456 & 3.763 & & & \\
\hline Thr & 7.865 & 3.963 & 3.882 & 0.928 & \\
\hline Alaninol & 7.961 & 3.886 & 0.898 & & H1/1': $3.758 / 3.602$ \\
\hline tert.Butyl & 8.066 & & & & $t \mathrm{Bu}: 0.997$ \\
\hline
\end{tabular}

Table 16: ${ }^{1} \mathrm{H}$ NMR chemical shifts of $\mathbf{8}$ (determined by ${ }^{1} \mathrm{H}, \mathrm{TOCSY}$, ROESY, COSY, HSQC). 


\begin{tabular}{|c|c|c|c|c|}
\hline Spin System & $\mathrm{C} \alpha$ & $C \beta$ & $\mathrm{C} \gamma$ & Miscellaneous \\
\hline ChinOA & & & & $\begin{array}{l}\text { C2: 146.766, C3: 121.091, C4: } 143.270, \mathrm{C} 5: 120.862, \mathrm{C} 6: \\
\text { 129.172, C7: } 113.125, \mathrm{O}-\mathrm{CH}_{2}: 66.853\end{array}$ \\
\hline $\begin{array}{l}\text { Lys } \\
\text { Val }\end{array}$ & $\begin{array}{l}53.003 \\
59005\end{array}$ & 29.602 & $\begin{array}{l}21.459 \\
17955\end{array}$ & C $\delta: 26.049$, Cع: 38.978 \\
\hline Gly & 41.646 & & & \\
\hline Thr & 59.005 & $44.509 ? ?$ & 18.251 & \\
\hline $\begin{array}{l}\text { Alaninol } \\
\text { tert.Butyl }\end{array}$ & 67.038 & 14.649 & & $\begin{array}{l}\text { C1: } 65.93 \\
t \text { Bu: } 27.48\end{array}$ \\
\hline
\end{tabular}

Table 17: ${ }^{13} \mathrm{C}$ NMR chemical shifts of 8 (determined from the HSQC).

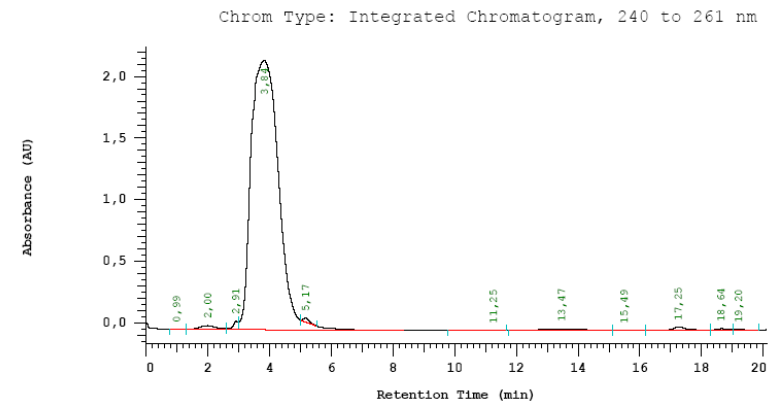

Figure 6 HPLC chromatogram of 8.

\section{Compound 9}

Compound 9 was synthesized in $5 \%$ yield over all steps $(1.9 \mathrm{mg}, 2.5 \mu \mathrm{mol})$

9 is a white solid, MALDI-TOF-MS: $756\left(\mathrm{M}+\mathrm{H}^{+}\right), 778\left(\mathrm{M}+\mathrm{Na}^{+}\right), 794\left(\mathrm{M}+\mathrm{K}^{+}\right)$.

NMR data: $700 \mathrm{MHz}, \mathrm{T}=285 \mathrm{~K}, \mathrm{pH}=2, \mathrm{H}_{2} \mathrm{O} / \mathrm{D}_{2} \mathrm{O}$ 9:1, calibrated to $\mathrm{HDO}=4.7 \mathrm{ppm}$ und Lys-C $\varepsilon=38.679 \mathrm{ppm}$

\begin{tabular}{|l|l|l|l|l|l|}
\hline Spin System & $\mathbf{N H}$ & $\mathbf{H} \alpha / \alpha^{\prime}$ & $\mathbf{H} \beta / \beta^{\prime}$ & $\mathbf{H} \gamma / \gamma^{\prime}$ & Miscellaneous \\
\hline$\beta-N O A$ & & & & & $\begin{array}{l}\text { H1: 7.010, H3: 7.059, H4: 7.672, H5: } \\
7.657, \mathrm{H} 6: 7.215, \mathrm{H} 7: 7.303, \mathrm{H} 8: 7.599, \\
\text { O-CH } 2: 4.638\end{array}$ \\
\hline Lys & 8.329 & 4.230 & $1.570 / 1.493$ & 0.909 & $\begin{array}{l}\mathrm{H} \delta: 1.256, \mathrm{H}: 2.345, \\
\text { NH-Z: 7.174 }\end{array}$ \\
\hline Val & 8.068 & 3.865 & 1.788 & 0.685 & \\
\hline Gly & 8.405 & 3.768 & & & \\
\hline Thr & 7.809 & 3.980 & 3.909 & 0.948 & \\
\hline Alaninol & 7.921 & 3.944 & 0.935 & & $\mathrm{H1} / 1^{\prime}: 3.839 / 3.704$ \\
\hline Cyclopentyl & 6.612 & & & & $\begin{array}{l}\mathrm{H} 1: 3.082, \mathrm{H} 2 / 2^{\prime}: 1.571 / 0.938, \\
\mathrm{H} 3 / 3^{\prime}: 1.441 / 1.061\end{array}$ \\
\hline
\end{tabular}

Table 18: ${ }^{1} \mathrm{H}$ NMR chemical shifts of 9 (determined by ${ }^{1} \mathrm{H}$, TOCSY, ROESY, COSY, HSQC).

\begin{tabular}{|l|l|l|l|l|}
\hline Spin System & $\mathrm{C} \alpha$ & $\mathrm{C} \beta$ & $\mathrm{C} \gamma$ & Miscellaneous \\
\hline$\beta-N O A$ & & & & $\begin{array}{l}\mathrm{C} 1: 106.644, \mathrm{C} 3: 117.551, \mathrm{C} 4: 129.345, \mathrm{C} 5: 126.878, \mathrm{C} 6: \\
123.325, \mathrm{C} 7: 126.73, \mathrm{C} 8: 126.73, \mathrm{O}_{-} \mathrm{CH}_{2}: 65.895\end{array}$ \\
\hline Lys & 52.300 & 29.605 & 21.486 & $\mathrm{C} 8: 25.641, \mathrm{C} \varepsilon: 38.679$ \\
\hline Val & 59.153 & 29.318 & 17.856 & \\
\hline Gly & 41.992 & & & \\
\hline $\begin{array}{l}\text { Thr } \\
\text { Alaninol }\end{array}$ & 58.986 & 66.898 & 18.238 & \\
\hline Cyclopentyl & 44.388 & 14.418 & & $\mathrm{C} 1: 65.839$ \\
\hline
\end{tabular}

Table 19: ${ }^{13} \mathrm{C}$ NMR chemical shifts of $\mathbf{9}$ (determined from the HSQC). 


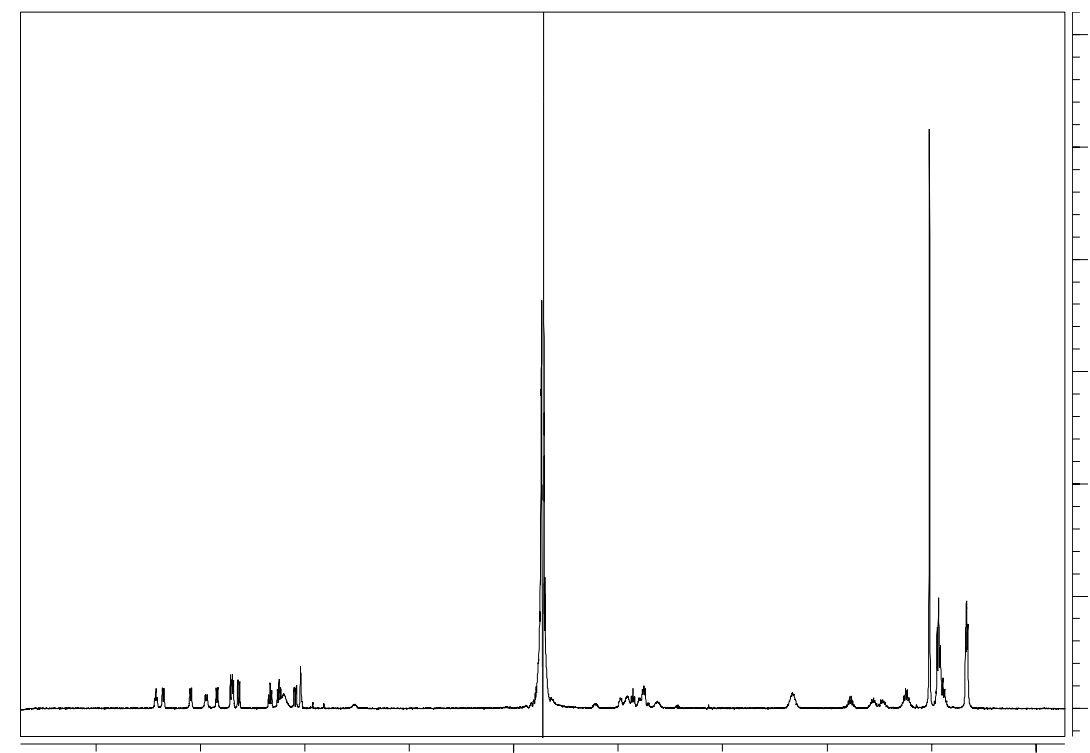

Figure 7: ${ }^{1} \mathrm{H}$ NMR WATERGATE spectrum of 9 in $\mathrm{D}_{2} \mathrm{O} / \mathrm{H}_{2} \mathrm{O}$ 1:9, $p \mathrm{H} 3$.

Compound 10

Compound 10 was synthesized in $4.9 \%$ yield over all steps ( $2 \mathrm{mg}, 2.4 \mu \mathrm{mol})$ 10 is a white solid, MALDI-TOF-MS: $822\left(\mathrm{M}+\mathrm{H}^{+}\right), 844\left(\mathrm{M}+\mathrm{Na}^{+}\right), 860\left(\mathrm{M}+\mathrm{K}^{+}\right)$.

NMR data: $500 \mathrm{MHz}, \mathrm{T}=285 \mathrm{~K}, \mathrm{pH}=2, \mathrm{H}_{2} \mathrm{O} / \mathrm{D}_{2} \mathrm{O}$ 9:1, calibrated to $\mathrm{HDO}=4.700 \mathrm{ppm}$ and Lys-C $\varepsilon=39.442 \mathrm{ppm}$

\begin{tabular}{|c|c|c|c|c|c|}
\hline Spin System & $\mathrm{NH}$ & $H \alpha / \alpha^{\prime}$ & $H \beta / \beta^{\prime}$ & $\mathrm{H} \gamma / \gamma^{\prime}$ & Miscellaneous \\
\hline$\beta$-NOA & & & & & $\begin{array}{l}\text { H1: 7.04, H3: 7.092, H4: 7.698, H5: } \\
\text { 7.690, H6: 7.241, H7: 7.329, H8: 7.632, } \\
\text { O-CH2: } 4.625\end{array}$ \\
\hline Lys & 8,340 & 4245 & $1.557 / 1.48$ & 0.913 & 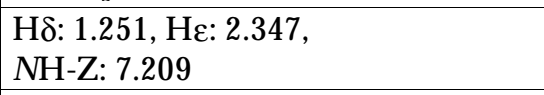 \\
\hline Val & 8.093 & 3.863 & 1.799 & 0.678 & \\
\hline Gly & 8.4330 & 3.774 & & & \\
\hline Thr & 7.828 & 3.990 & 3.914 & 0.952 & \\
\hline $\begin{array}{l}\text { Alaninol } \\
\text { Adamantyl }\end{array}$ & $\begin{array}{l}7.910 \\
6.828\end{array}$ & 3.939 & 0.958 & & $\begin{array}{l}\mathrm{H} 1 / 1^{\prime}: 3.793 / 3.634 \\
\mathrm{H} 2 / 2^{\prime}: 1.672 / 1.607, \mathrm{H} 3: 1.82, \mathrm{H} 4 / 4^{\prime}: \\
1.431\end{array}$ \\
\hline
\end{tabular}

Table 20: ${ }^{1} \mathrm{H}$ NMR chemical shifts of $\mathbf{1 0}$ (determined by ${ }^{1} \mathrm{H}$, TOCSY, ROESY, COSY, HSQC).

\begin{tabular}{|l|l|l|l|l|}
\hline Spin System & $\mathrm{C} \alpha$ & $\mathrm{C} \beta$ & $\mathrm{C} \gamma$ & Miscellaneous \\
\hline$\beta-N O A$ & & & & $\begin{array}{l}\text { C1: } 107.093, \mathrm{C} 3: 118.770, \text { C4: 127.839, C5: 127.820, C6: } \\
124.891, \mathrm{C7}: 127.499, \mathrm{C} 8: 127.215\end{array}$ \\
\hline Lys & 53.250 & 30.392 & 16.028 & $\mathrm{C} 8: 26.634, \mathrm{C} \varepsilon: 39.442$ \\
\hline Val & 60.262 & 29.358 & 18.462 & \\
\hline Gly & 42.765 & & & \\
\hline Thr & 59.940 & 67.531 & 16.26 & \\
\hline Alaninol & 45.274 & 16 & 66.566 & \\
\hline Adamantyl & & & & $\mathrm{C} 2: 41.300, \mathrm{C} 3: 29.592, \mathrm{C} 4: 35.965$ \\
\hline
\end{tabular}

Table 21: ${ }^{13} \mathrm{C} N M R$ chemical shifts of $\mathbf{1 0}$ (determined from the HSQC).

$\mathrm{S} 10$ 


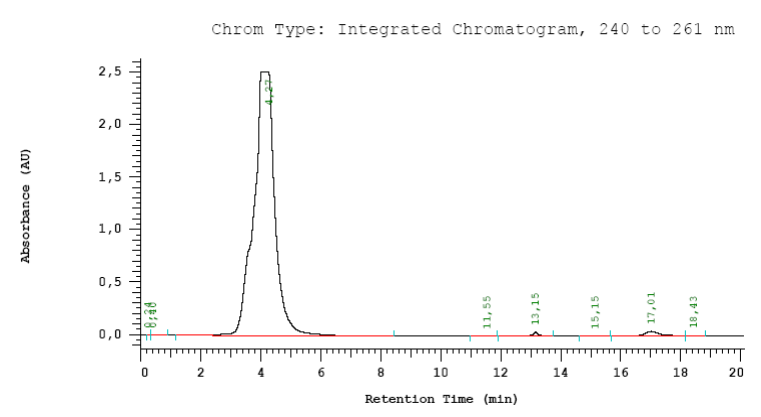

Figure 8: HPLC chromatogram of $\mathbf{1 0 .}$

\section{Compound 11}

Compound 11 was synthesized in $5.1 \%$ yield over all steps (1.9 mg, $2.6 \mu \mathrm{mol})$

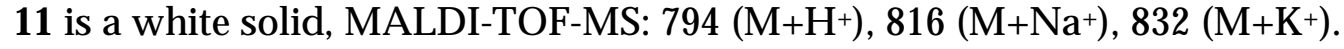

NMR data: $500 \mathrm{MHz}, \mathrm{T}=285 \mathrm{~K}, \mathrm{pH}=2, \mathrm{H}_{2} \mathrm{O} / \mathrm{D}_{2} \mathrm{O}$ 9:1, calibrated to $\mathrm{HDO}=4.700 \mathrm{ppm}$ and Lys-C $\varepsilon=39.412 \mathrm{pm}$

\begin{tabular}{|c|c|c|c|c|c|}
\hline Spin System & $\mathrm{NH}$ & $H \alpha / \alpha^{\prime}$ & $H \beta / \beta^{\prime}$ & $\mathrm{H} \gamma / \gamma^{\prime}$ & Miscellaneous \\
\hline$\beta$-NOA & & & & & $\begin{array}{l}\mathrm{H} 1: \text { 7.023, H3: 7.076, H4: 7.692, H5: } \\
\text { 7.673, H6: 7.232, H7: 7.320, H8: 7.620, } \\
\text { O-CH2: } 4.613\end{array}$ \\
\hline Lys & 8.338 & 4.236 & $1.527 / 1.457$ & 1.218 & 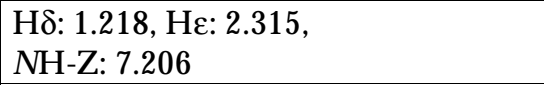 \\
\hline Val & 8.079 & 3.856 & 1.759 & 0.655 & \\
\hline Gly & 8.412 & 3.750 & & & \\
\hline Thr & 7.828 & 3.982 & 3.898 & 0.922 & \\
\hline Alaninol & 7.926 & 3.919 & 0.901 & & H1/1': $3.771 / 3.624$ \\
\hline tert.-Butyl & 6.915 & & & & $t \mathrm{Bu}: 1.009$ \\
\hline
\end{tabular}

Table 22: ${ }^{1} \mathrm{H}$ NMR chemical shifts of $\mathbf{1 1}$ (determined by ${ }^{1} \mathrm{H}, \mathrm{TOCSY}, \mathrm{ROESY}, \mathrm{COSY}, \mathrm{HSQC}$ ).

\begin{tabular}{|l|l|l|l|l|}
\hline $\begin{array}{l}\text { Spin System } \\
\beta-N O A\end{array}$ & $\mathrm{C} \alpha$ & $\mathrm{C} \beta$ & $\mathrm{C} \gamma$ & $\begin{array}{l}\text { Miscellaneous } \\
\text { C1: 107.161, C3: 118.654, C4: 128, C5: 128, C6: 124.855, } \\
\mathrm{C} 7: 127.415, \mathrm{H}: 127.131\end{array}$ \\
\hline Lys & 53.297 & 30.748 & 22.180 & $\mathrm{C} \delta: 26.633, \mathrm{C} \varepsilon: 39.412$ \\
\hline Val & 60.160 & 30.312 & 18.114 & \\
\hline $\begin{array}{l}\text { Gly } \\
\text { Thr }\end{array}$ & 42.720 & & & \\
\hline Alaninol & 59.823 & 67.530 & 19.131 & \\
\hline tert.-Butyl & & & & $\mathrm{C} 1: 67.024$ \\
\hline
\end{tabular}

Table 23: ${ }^{13} \mathrm{C} N M R$ chemical shifts of $\mathbf{1 1}$ (determined from the HSQC) 
Chrom Type: Integrated Chromatogram, 240 to $261 \mathrm{~nm}$

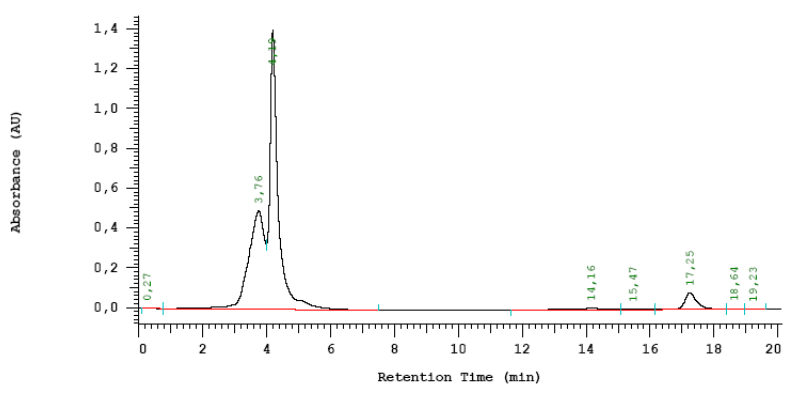

Figure 9: HPLC chromatogram of 11. Two peaks are present in the chromatogram due to elution of the protonated and unprotonated species. Only the mass of the desired product is detectable in the MALDI-TOF. 


\section{c) Surface plasmon resonance}

Experimental details and data points fitted to a one-site binding model (Figure 1). SPR experiments

The SPR data were determined on a BIACORE 3000 instrument using CM5 chips. $106 \mathrm{fmol}$ and $220 \mathrm{fmol}$, respectively, SCD4 were covalently attached to the activated dextrane matrix. The surface was activated by $\mathrm{N}$-hydroxy succinimide and $\mathrm{N}$-(3-dimethylaminopropyl)-N'-ethylcarbodiimide hydrochloride for the coupling of the protein. After coupling of the protein the matrix wascapped with ethanolamine. The activity of the immobilized protein was checked by gp120 binding; extrapolation of the data showed a n activity of 50\% (53 fmol) and $20 \%$ (44 fmol), respectively, of the immobilized CD4..$^{[2]} \mathrm{SCD} 4$ was obtained from Progenics Phamaceuticals, Tamytown, New York, USA. Catalogue-No.: PRO 1008-1, Lot 48 (recombinant human soluble CD4 $(\mathrm{CHO})$, purity $>95 \%$, a mino acids $1-370$ of the natural CD4 (45 kDa)), gp120 was purchased from the National Institute for Biological Standards and Control, catalogue-No. EVA607, http://www.nibsc.ac.uk/catalog/a ids-reagent (recombinant HIV-1 IIIB GP120, 120 kDa. purity $>95 \%$, expression system: baculovinus). The KD value of the gp120/CD4 interaction is in the low $\mathrm{nm}$ range (depending on the method). ${ }^{[3]}$ The acitivity was calculated following equations 1 and 2 .

$$
\begin{array}{ll}
\operatorname{activity}(C D 4)=\frac{R U[g p 120] \max _{\text {measured }}}{R U[g p 120] \max _{\text {calculated }}} & \text { equation 1 } \\
\text { activeCD4[fmol] }=\text { acitivity }(C D 4) * \text { immobilizedCD4[fmol }] & \text { equation 2 }
\end{array}
$$

We used a dilution series with concentrations of the peptid o mimetic s of 4,6 , $8,10,12,16,20,25,30,50,100,500$ and $1000 \mu M$ in HBS-EP (10 mM HEPES, 150 mM $\mathrm{NaCl}, 3 \mathrm{mM}$ EDTA, $0.005 \%$ surfactant P20, pH 7.4) buffer. Contact time was $120 \mathrm{~s}$ with a flow rate of $15 \mu \mathrm{L} / \mathrm{min}$ and a dissociation time of $60 \mathrm{~s}$. Every concentration 
was measured twice starting from the lowest concentration. The surface was regenerated with two injections of $100 \mathrm{mM} \mathrm{H}_{3} \mathrm{PO}_{4}$ for $32 \mathrm{~s}$ with the same flow rate. The dependence of the response units (RU; $1 R U \sim 1 \mathrm{pg}$ ) on the concentration can be used to obta in $\mathrm{K}_{\mathrm{D}}$ values using eq. 3 :

$$
R U([c])=\frac{[c]^{*} R U_{\max }}{K_{D}+[c]}
$$

\section{equation 3}

The peptidomimetics did not show an asymptotic behavior at high concentrations in the Biacore assay. This may be due to unspecific binding of the ligands to the protein surface or because of a second binding site with a lower binding affinity. Therefore, only data points representing a typical saturable association curve were used for data analysis.

Data points fitted to a one-site binding model:
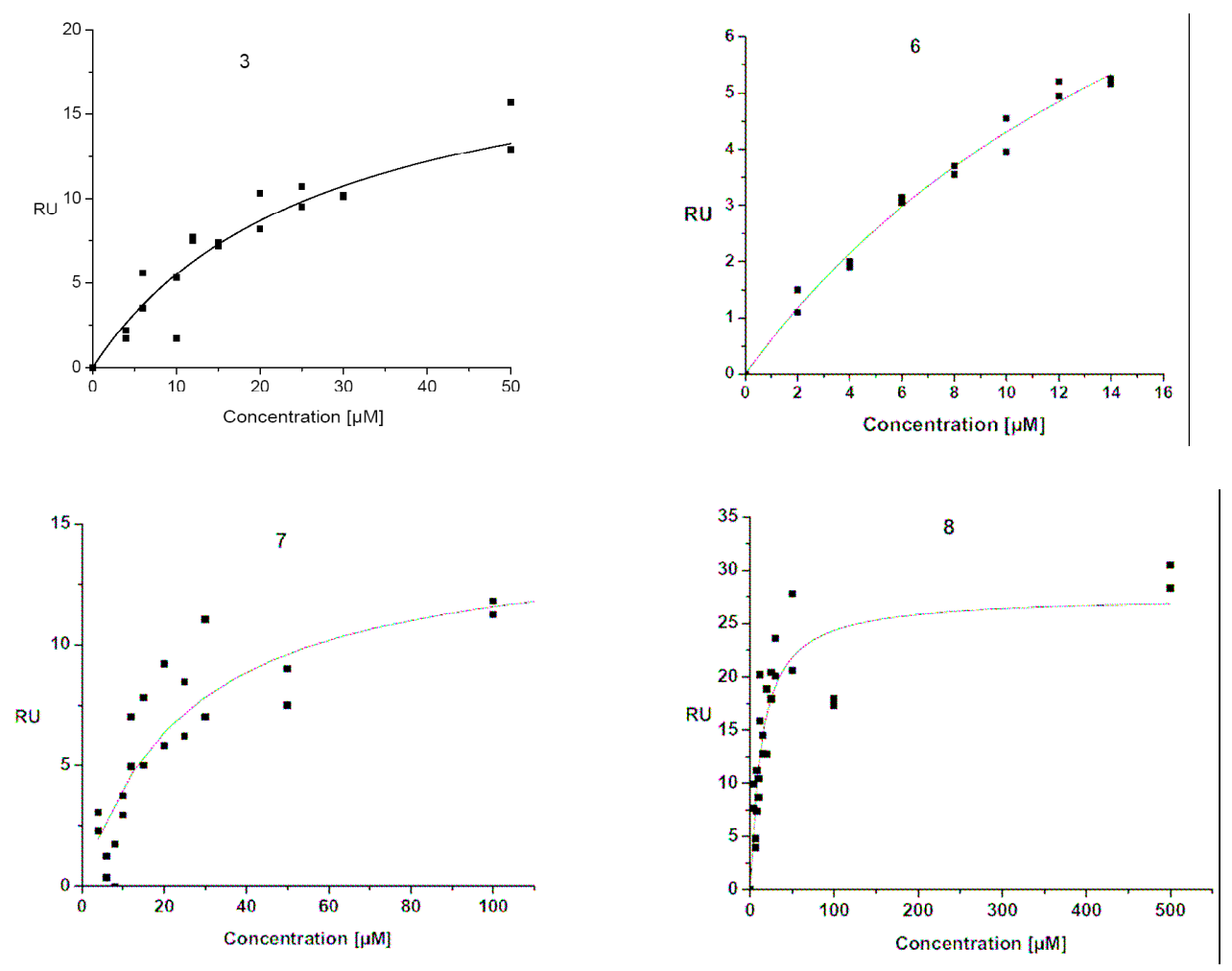

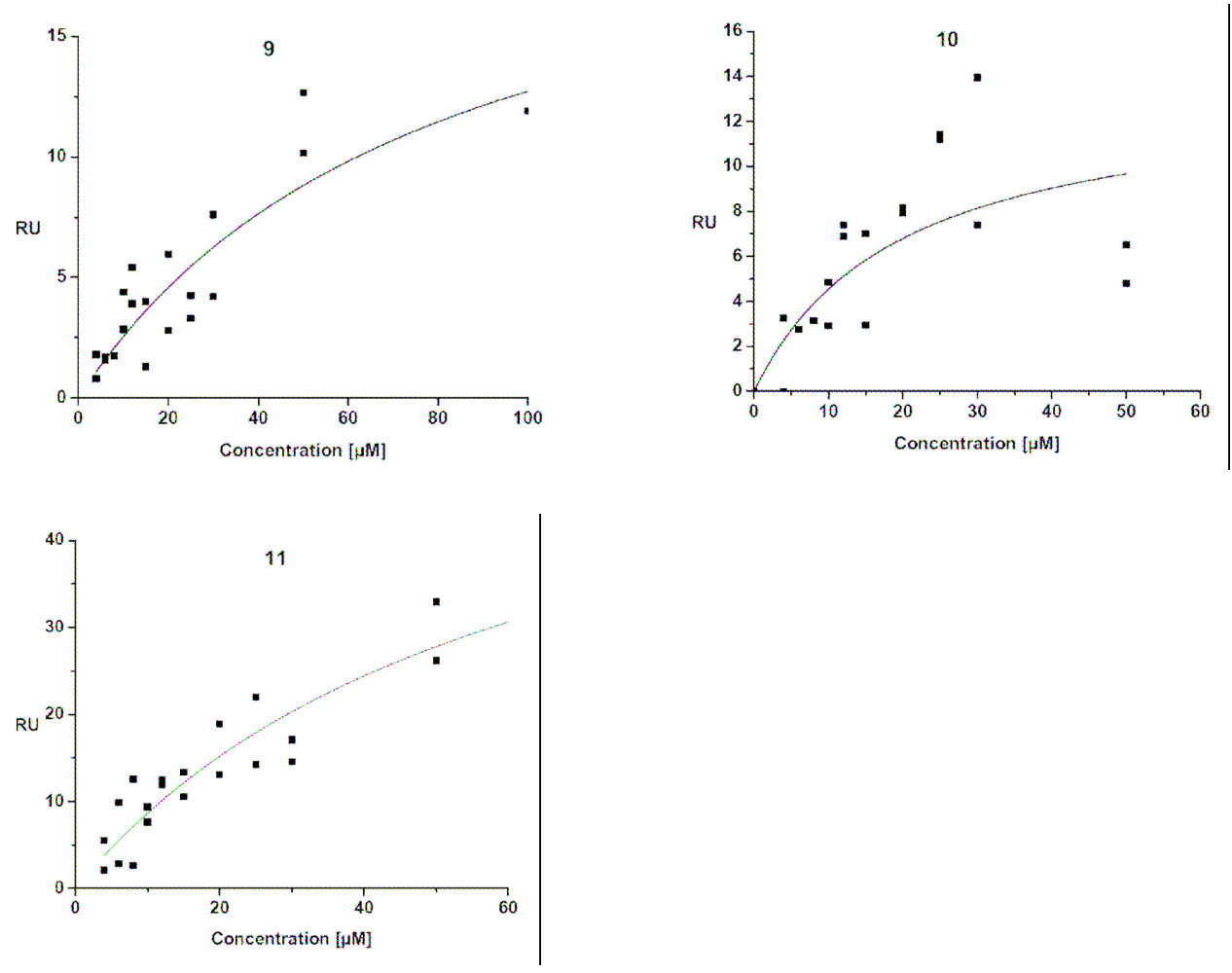

Figure 10: Data points of the SPR experiments fitted to a one-site binding model (equation 3). 


\section{d) STD NMR experiments}

Experimental details and spectra for 6 (Figure 11).

Preparation of the samples:

Commerc ially available SCD4 (see above) $(200 \mu \mathrm{g}, 4.4 \mathrm{nmol})$ was dissolved in buffer ( $200 \mu \mathrm{L} \mathrm{D}_{2} \mathrm{O}$, containing $8 \mathrm{mM} \mathrm{Na}_{2} \mathrm{HPO}_{4}, 2 \mathrm{mM} \mathrm{NaH} \mathrm{PO}_{4}, 140 \mathrm{mM} \mathrm{NaCl}, 3 \mathrm{mM}$ $\mathrm{KCl}$ and $6 \mathrm{mM} \mathrm{NaN}{ }_{3}$, adjusted to $\mathrm{pH}=7-7.5$ (not corrected) with $0.1 \mathrm{M} \mathrm{DCl}$ ). Through repeated dilution and membrane centrifugation, the sample was rebuffered in deuterated PBS, resulting in 250-300 $\mu \mathrm{L}$ solution (exac tly measured by weighing). The protein solution was split into two halves and $5 \mu \mathrm{L} 1 \mathrm{mM}$ DSS solution in $\mathrm{D}_{2} \mathrm{O}$ was added to each portion. The samples were filled to $250 \mu \mathrm{L}$ each, one with buffer and the other one with buffer containing $\mathbf{4}$ or $\mathbf{6}$, respectively, to produce the highest concentrations of $418 \mu \mathrm{M}$ (4) and $324 \mu \mathrm{M}$ (6), respectively. The samples were transferred into Shigemi NMR tubes and measured on a Bruker Avance $700 \mathrm{MHz}$ spectrometer with a cryo probe. Other concentrations of the ligand were achieved by diluting the ligand solution with the buffered protein solution from the other sample and vice versa. The exact concentrations of the ligand were determined by comparison with the intemal DSS standard. The on resonance frequency was detemined by measuring the ligand sample only. At $1.0 \mathrm{ppm}$ (for 7) and $-1.5 \mathrm{ppm}$ (for 4), all discoverable artefacts were $<1$ rel. STD\%, respectively. The number of scans of the reference spectra and the STD spectra was adjusted to the concentration and was up to $32 \mathrm{k}$ for the STD spectrum at the lowest ligand concentration of $48 \mu \mathrm{M}$ for ligand 4 , and $33 \mu \mathrm{M}$ for ligand 7 , respectively. The off resonance frequency was set to $28.6 \mathrm{ppm}$.

Data a nalysis:

$1 \mathrm{D}^{\mathrm{I}} \mathrm{H}$ NMR spectra were acquired at a spectral width of $10 \mathrm{ppm}$ and with $32 \mathrm{k}$ data points. Before Fourier transformation, these were zero filled to 64k. Through S 16 
multiplication of the FID with an exponential function (line broadening: $5 \mathrm{~Hz}$ ), an increase of the signal noise ratio was achieved, and subsequently the phase was corrected. A Tlp filter of 15 ms with an attenuation of $12 \mathrm{~dB}$ eliminated nearly all protein signals. The phase cycle of the STD experiments was selected, such that the subtraction needed for the difference spectra occurred on altemating scans. In this way, artifacts due to temperature or magnetic inhomogeneities are minimized. On- and off-resonance frequency of the presaturation pulses altemated each scan. All STD experiments were recorded with WATERGATE water suppression. For detemination of the relative STD\% and the STD amplification factor, a reference experiment wasrecorded with the same conditions, processed and phased with the same parameters. The integrals were compared in the dual display mode in XWINNMR (version 3.1, Bruker). The temperature of the samples was $285 \mathrm{~K}$.

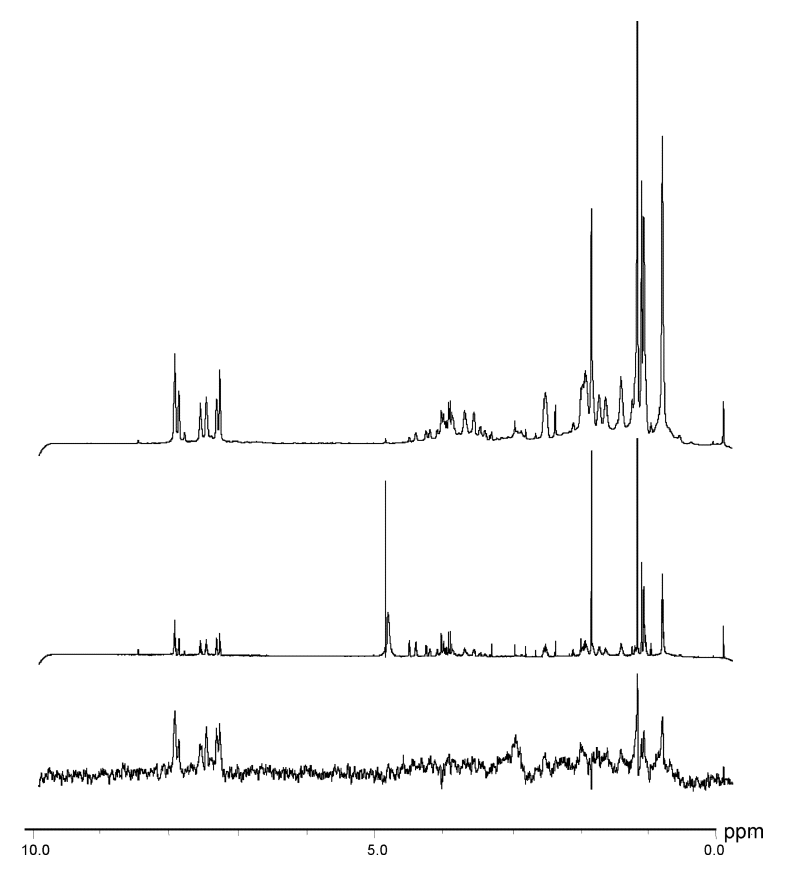

Figure 2: (top) ${ }^{1} \mathrm{H}$ NMR spectrum of a mixture of $\mathbf{6}$ and CD4; (middle) ${ }^{1} \mathrm{H}$ NMR spectrum of $\mathbf{6}$; (bottom) ${ }^{1} \mathrm{H}$ STD NMR spectrum of 6 when binding to CD4. 


\section{e) Prona se Digestion Assay}

A solution of $0.8 \mu \mathrm{g}$ pronase from streptomyces griseus in $100 \mu \mathrm{L} 0.1 \mathrm{M}$ Tris- $\mathrm{HCl}$ buffer, $\mathrm{pH}$ 7-7.5 was mixed with $100 \mu \mathrm{L}$ of a $200 \mu \mathrm{M}$ solution of the respective peptide or peptidomimetic in $\mathrm{D}_{2} \mathrm{O}$. After incubation at $37^{\circ} \mathrm{C}$, samples of $20 \mu \mathrm{L}$ were taken after 5,10 , 15,20 , and 30 minutes and directly pipetted into $40 \mu \mathrm{L} 1 \mathrm{M}$ acetic acid. The mixtures were

vortexed, centrifuged, and frozen in liquid nitrogen till further analysis. The thawed mixtures were applied directly to a RP-HPLC system, with UV-VIS detection at $280 \mathrm{nM}$. 


\section{f) Docking Studies}

Docking studies were carried out on Silicon Graphics Octane or Octane2 computers using the program Flexidock of the Sybyl (version 6.9) software package (Tripos, Inc.). The structure of CD4 was obtained from a Protein Data Bank file (code 1gc1.pdb) ${ }^{[4]}$ and was treated as described before. ${ }^{[1]}$ The force field calculations take into account van der Waals, electrostatic, torsional, and constraint energy terms. All bonds of the ligand were freely rotatable during the docking. At the receptor, only the amino acids of the CD4 forming the binding pocket (identified as amino acids $\mathrm{Q}_{40} \mathrm{GSF}_{43}, \mathrm{R}_{59} \mathrm{SLWDQG}_{65}$, and $\mathrm{S}_{23}$ ) had rotatable bonds, to allow a partial induced fit. Docking with Flexidock is performed with a genetical algorithm. Docking for each compound was performed in cycles of 50000 generations, after which the docked structures and energies were evaluated. No further docking was conducted when the binding mode and calculated binding affinity did not change in comparison to a previous docking cycle. 
g) references

1: Neffe, A.T.; Bilang, M.; Meyer, B. Synthesis and optimization of peptidomimetics as HIV entry inhibitors against the receptor protein CD4 using STD NMR and ligand docking. Org. Biomol. Chem. 2006, 4, 3259-3267.

2: Wu, H.; Myszka, D.G.; Tendian, S.W.; Brouilette, C.G.; Sweet, R.W.; Chaiken, I.M.; Hendrickson, W.A. Kinetic and structural analysis of mutant CD4 receptors that are defective in HIV gp120 binding. Proc. Natl. Acad. Sci. USA 1996, 93, 15030-15035.

3: S.M. Schnittman, H.C. Lane, J. Roth, A. Burrows, T.M. Folks, J.H. Kehrl, S. Koenig, P. Berman, A.S. Fauci, Characterization of GP120 binding to CD4 and an assay that measures ability of sera to inhibit this binding. J. Immunol. 1988, 141, 4181-4186.

4: P.D. Kwong, R. Wyatt, J. Robinson, R.W. Sweet, J. Sodroski, W.A. Hendrickson, Structure of an HIV gp120 envelope glycoprotein in complex with the CD4 receptor and a neutralizing human antibody. Nature 1998, 393, 648-659. 